\title{
ASOCIACIÓN DE ROTAVIRUS Y Escherichia coli FIMBRIADA COMO AGENTES CAUSALES DE INFECCIONES ENTÉRICAS EN ALPACAS NEONATAS
}

\author{
Relationship Between rotavirus and Fimbriated Escherichia coli in ENTERIC \\ INFECTIONS OF NEONATAL AlPaCAS
}

\author{
Siever Morales C. ${ }^{1,2}$, Daniel Paredes L. ${ }^{3}$ y Danilo Pezo C. ${ }^{4}$
}

Resumen

\begin{abstract}
El objetivo del presente trabajo fue determinar la presencia y la asociación entre rotavirus y la Escherichia coli fimbriada como agentes patógenos causantes de las infecciones entéricas en condiciones naturales en alpacas neonatas. La detección de rotavirus y de antígenos fimbriales se realizó mediante aglutinación con anticuerpos monoclonales embebidos en látex y la biotipificación se realizó mediante el sistema API 20E. En el $9.3 \%$ de las muestras de heces se detectó rotavirus. Se encontró 10 biotipos de E. coli, donde se detectó un $26 \%$ de antígeno F41 en alpacas neonatas con infección entérica en contraste con el $48 \%$ en los aislamientos de E. coli de alpacas clínicamente sanas. Rotavirus se encuentra en asociación con el $18.8 \%$ de los aislamientos de E. coli identificados como biotipos A y C. El 57.8\% de aislamientos de E. coli de alpacas neonatas con infección entérica que contienen antígeno fimbrial F41 se encuentran en conjunto con la presencia de rotavirus. Por lo tanto, la asociación entre rotavirus, el biotipo de $E$. coli y el antígeno fimbrial F41 podría ser la causa de diarrea en el 18.8\% del 30-40\% de la población de alpacas afectadas.
\end{abstract}

Palabras clave: E. coli, rotavirus, diarrea, alpaca

\section{Abstract}

The objective of the present research was to determine the presence and the association between rotavirus, biotype E. coli and fimbriated E. coli in the enteric infections of neonatal alpacas under natural conditions. The detection of rotavirus was carried out by agglutination with monoclonal antibodies, the biotyping of $E$. coli by API $20 \mathrm{E}$ system and the detection of fimbrial antigens by agglutination using monoclonal antibodies. In 15 out of 160 (9.4\%) of fecal samples from neonatal alpacas with enteric infection was detected rotavirus. Ten biotypes of $E$. coli was found and $26 \%$ of the $E$. coli isolates from alpaca neonates with enteric infection had the F41 antigen in contrast with $48 \%$ from clinically healthy neonatal alpacas. Rotavirus was found in association

${ }^{1}$ Laboratorio de Microbiología y Parasitología Veterinaria, ${ }^{4}$ Laboratorio de Microbiología, Estación Experimental del Centro de Investigaciones IVITA - Maranganí, Facultad de Medicina Veterinaria, Universidad Nacional Mayor de San Marcos, Lima

${ }^{2}$ E-mail: smoralesc@unmsm.edu.pe

${ }^{3}$ Área de Sanidad Animal, Departamento de Ciencias Pecuarias, Universidad Nacional Agraria de la Selva, UNAS, Tingo María 
with $18.8 \%$ of the $E$. coli isolates identified as biotypes A and C. The $57.8 \%$ of E. coli isolates from neonatal alpacas with enteric infections which contained the fimbrial antigen F41 were found in association with rotavirus. In the present research has been found that under natural conditions, rotavirus is present in conjunction with a different biotype of $E$. coli and fimbrial antigens $\mathrm{F} 41$ in $18.8 \%$; therefore, the association between rotavirus, E. coli biotype and fimbrial antigen F41 may be the cause of diarrhea in $18.8 \%$ of $30-40 \%$ of neonatal alpacas affected.

Key words: $E$. coli, rotavirus, diarrhea, alpaca

\section{INTRODUCCIÓN}

La Escherichia coli enterotoxigénica y enteropatogénica, así como los rotavirus, son considerados agentes causales del complejo diarreico en neonatos de la mayoría de especies de animales domésticos. También se ha detectado que la presencia de $E$. coli patogénica se distribuye en biotipos diferentes de acuerdo a la especie en donde se halla ubicada. En estos biotipos se han encontrado factores de virulencia tales como estructuras fimbriales (F4, F5, F6 y F41) y la producción de toxinas (Ramirez et al., 2000). Por otro lado, los rotavirus patógenos de las diferentes especies animales se han agrupado en patotipos designados como grupo A, $\mathrm{B}, \mathrm{C}$ y D, donde el grupo A es patógeno para la mayoría de las especies de animales domésticos (Fodha et al., 2005).

Se ha descrito que la infección previa o simultánea por rotavirus u otros virus entéricos realizados en forma experimental permiten la colonización de E. coli en el intestino de terneros y cerdos lactantes. Las lesiones en el intestino son más extensas y severas en coinfecciones entericas por ambos agentes, por lo que las diarreas son más consistentes que en cualquiera de las infecciones por separado (Benfield et al., 1988). Existen pocos reportes sobre la prevalencia de anticuerpos contra rotavirus en alpacas (Lama pacos), llamas (Lama glama) y guanacos (Lama guanicoe) (Puntel et al., 1999; Parreño et al., 2001); sin embargo, no se dispone de publicaciones sobre la detec- ción y el rol que juega la $E$. coli fimbriada y los rotavirus en el desarrollo del complejo diarreico de las alpacas neonatas en forma natural.

La presente investigación tuvo como objetivo determinar la presencia y la asociación entre rotavirus y la Escherichia coli fimbriada como agentes patógenos causantes de las infecciones entéricas en alpacas neonatas.

\section{MATERIALeS y Métodos}

Se trabajó con alpacas nacidas entre enero y marzo del 2004, en la Estación Experimental de Camélidos Sudamericanos de la Universidad San Antonio Abad del Cusco, ubicado en el abra La Raya-Maranganí.

Se colectaron muestras de heces mediante hisopados rectales en 320 alpacas de 1 a 30 días de edad. De éstas, 160 fueron de alpacas neonatas clínicamente sanas y 160 de alpacas con cuadro de diarrea aguda o crónica. Las muestras se procesaron en forma inmediata a su recolección, donde cada uno de los aislados bacterianos con capacidad de fermentar lactosa se procesaron utilizando el kit de API-20E (Laboratorio Bio Merieux, Francia) de acuerdo a lo descrito por Swanson y Collins (1980). Además, se evaluaron, vía anticuerpos monoclonales, los tipos de antígenos fimbriales F4 (K88), F5(K99), F6(987P) y F41 en cada uno de doscientos aislamientos de $E$. coli. 
La detección del antígeno de rotavirus se efectuó mediante aglutinación con partículas de látex sensibilizados con un grupo específico de anticuerpos monoclonales contra rotavirus (Slidex Rota - kit 2, Biomerieux, Francia).

\section{Resultados y Discusión}

Se encontró el 9.3\% (15/160) de muestras positivas a rotavirus en el grupo de crías de alpacas con infección entérica (AIE), donde el 33.3\% (5/15) de antígenos de rotavirus se ubicaron en el biotipo A y el 66.7\% (10/15) se ubicaron en le biotipo C; mientras que todas las muestras de crías de alpacas clínicamente sanas (ACS) fueron negativas al antígeno de rotavirus.

Del agrupamiento de los aislamientos de E. coli basados en las respuestas frente a los sustratos bioquímicos del sistema API-20E, se obtuvieron diez biotipos (A-J). El 20 (16/80), $40(32 / 80), 25(20 / 80)$ y $20 \%$ (16/80) de los aislamientos se identificaron como biotipo $\mathrm{C}$, A, H y otros, respectivamente. El 26\% (26/ 100) de los aislamientos de las AIE expresaron antígenos solamente contra la fimbria F41, donde 19.3 (5/26), $38.5(10 / 26)$ y $26.9 \%$ (7/ 26) se ubicaron en los biotipos A, C y H, respectivamente (Fig. 1). En el grupo de ACS, el
$48 \%$ (48/100) de los aislados expresaron antígenos fimbriales F41, 8\% (8/100) en asociación doble de F6 y F41, y 2\% (2/100) en asociación triple de F4, F6 y F41.

Rotavirus se encontraron en asociación con el $12.5 \%$ (10/80) de los aislamientos de E. coli perteneciente al biotipo $\mathrm{C}$ y con el $6.3 \%(5 / 80)$ del biotipo A. En relación con antígenos fimbriales, el 38\% (10/26) de aislados de $E$ coli fueron del biotipo C y $19.3 \%$ (5/25) del biotipo A.

La infección entérica producida por la asociación entre rotavirus y $E$. coli llega a producir altos índices de mortalidad en animales y niños neonatos, si es que no se recurre a terapias apropiadas; sin embargo, en alpacas neonatas no se evidencia la misma tendencia y, más aún, si el proceso de recuperación se produce sin administración de fármacos antídotos. En la actualidad, no hay publicaciones disponibles de la interacción entre E. coli y rotavirus; no obstante, se dispone de información relacionada a los efectos que producen la asociación de estos agentes en forma experimental, indicando que se producen lesiones y diarreas más severas en el intestino que cuando actúa cada uno en forma separada (Runnels et al., 1986; Benfield et al., 1988). Además, siempre es bueno tener en cuenta que, usualmente, en

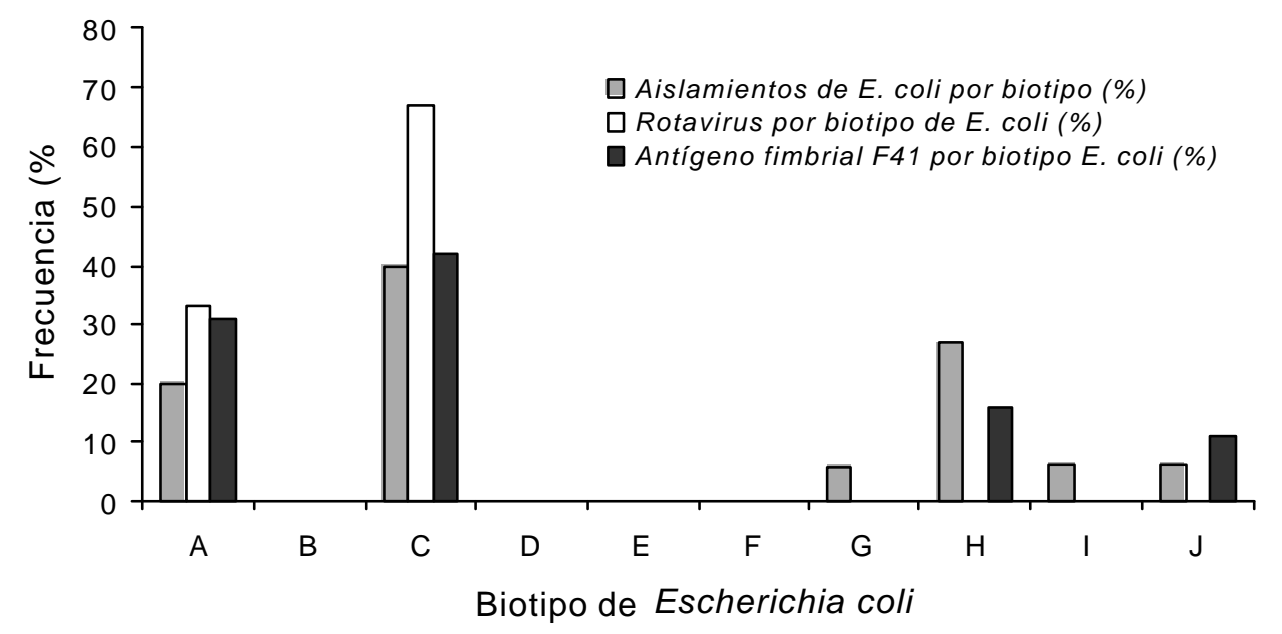

Figura 1. Asociación entre la frecuencia de rotavirus, el biotipo de E. coli y antígeno fimbrial F41 en alpacas neonatas con infección entérica 
los resultados de los trabajos experimentales no se toma en consideración el medio ambiente y la genética de los animales, factores que influyen en la presentación de una enfermedad.

Por lo tanto, este complejo diarreico en las alpacas neonatas deja muchas interrogantes, como por ejemplo, investigar otras características asociadas con la virulencia de E. coli (Vanderkerchove et al., 2005) y otros agentes microbianos relacionados como coronavirus, astrovirus, calicivirus (Williams y Barker, 2002), así como los mecanismos de inmunidad desarrollados por esta especie para controlar el ataque de estos patógenos.

\section{Conclusiones}

- Los rotavirus en condiciones naturales se encuentran conjuntamente con biotipos A y $\mathrm{C}$ de E. coli y antígenos fimbriales F41 en el 18.8\% de los aislados de E. coli.

- La asociación entre rotavirus, el biotipo de la $E$. coli, y el antígeno fimbrial F41 podría ser la causa de diarrea en el $18.8 \%$ del $30-40 \%$ de la población de alpacas neonatas afectadas.

\section{Agradecimientos}

A la Fundación Internacional para Ciencias (IFS), Suecia, por financiar el presente trabajo (Grant B-3296-1), a los doctores Hermelinda Rivera y Alberto Manchego, así como al personal de la Estación del IVITAMaranganí y de la Estación La Raya de la UNSAAC por el apoyo brindado durante el desarrollo del presente trabajo.

\section{Literatura Citada}

1. Benfield DA, Francis DH, Mc Adaragh JP, Johnson DD, Bergeland ME, Rossow K, Moore R. 1988.
Combined rotavirus and K99 Escherichia coli infection in gnobiotic pigs. Am J Vet Res 49: 330-337.

2. Fodha I, Boumaiza A, Chouikha A, Dewar J, Armah G, Geyer A, Trabelsi $A$, Steele AD. 2005. Detection of group A rotavirus strains circulating in calves in Tunisia. J Vet Med B 52: 49-50.

3. Parreño $V$, Constantini $V$, Cheetham S, Blanco Viera J, Saif LJ, Fernández F, Leoni L, Schudel A. 2001. First isolation of rotavirus associated with neonatal diarrhea in guanacos (Lama guanicoe) in the Argentinean Patagonia region. J Vet Med B 48: 713-720.

4. Puntel M, Fondevilla NA, Blanco Viera J, O'Donnell VK, Marchovecchio JF, Carrillo BJ, Schudel AA. 1999. Serological survey of viral antibodies in llamas (Lama glama) in Argentina. J Vet Med B 46: 157-161.

5. Ramirez A, Elli RP, Wilson RA. 2000. Escherichia coli piliada no atoxigénica de procesos diarreicos en crías de alpacas. En: II Congreso Mundial Sobre Camélidos. Cusco, Perú.

6. Runnels PL, Moon HW, Mathews PJ. 1986. Effects of microbial and host variables on the interaction of rotavirus and Escherichia coli infections in gnotobiotic calves. Am J Vet Res 47: 1542-1550.

7. Swason EC, Collins MT. 1980. Use of the API 20E system to identify veterinary Enterobactereacea. J Clin Microbiol 12: 10-14.

8. Vandekerchove D, Vandemaele F, Adriaensen $C$, Zaleska $M$, Hernalsteens JP, Baets LD, Butaye P, Van Immerseel $F$, Wattiau $P$, Laevens H, Mast J, Goddeeris B, Pasmans F. 2005. Virulence-associated traits in avian Escherichia coli: comparison between isolates from colibacillosisafected and clinically healthy layer flocks animals. Vet Microbiol 108: 75-87.

9. Williams ES, Barker IK. 2002. Infectious diseases of wild mammals. IOWA: Iowa University Press. 537 p. 